\title{
Adherence to the guidelines and the pathological diagnosis of high-risk gastrointestinal stromal tumors in the real world
}

\author{
Toshirou Nishida $^{1} \cdot$ Yoshiharu Sakai $^{2} \cdot$ Masakazu Takagi $^{3} \cdot$ Masato Ozaka $^{4} \cdot$ Yuko Kitagawa $^{5} \cdot$ Yukinori Kurokawa $^{6}$.

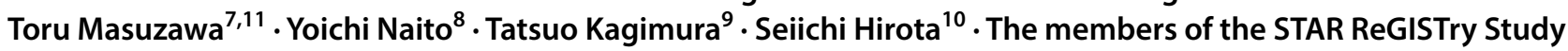 \\ Group
}

Received: 17 February 2019 / Accepted: 15 April 2019 / Published online: 30 April 2019

(c) The Author(s) 2019

\begin{abstract}
Background A multidisciplinary approach based on guidelines and pathological diagnosis by specialized pathologists are important for improving the prognosis and QoL of GIST patients. This study examined the adherence to the guidelines and the concordance of the pathological diagnosis of high-risk GISTs.

Patients and methods Among 541 patients with high-risk GISTs recruited to the prospective registry between Dec. 2012 and Dec. 2015, 534 patients were analyzed after central pathology with KIT and DOG1 IHC and genotyping of KIT and PDGFRA. Results Of the 534 patients, $432(81 \%)$ received imatinib adjuvant therapy at a starting dose of 400 or $300 \mathrm{mg} /$ day. Multivariate analysis indicated that age (HR 0.71; 95\% CI 0.58-0.88), tumor size (HR for $>10 \mathrm{~cm}$ vs $<5 \mathrm{~cm}, 3.87 ; 95 \%$ CI 1.72-8.74), mitosis (HR for $>10$ vs $<5,3.54$; 95\% CI 1.84-6.79), tumor rupture (HR 3.69; 95\% CI 1.43-9.52) and performance status (HR 0.55; 95\% CI 0.31-0.99) were independently related to adjuvant therapy. Among the 534 high-risk GISTs diagnosed locally, 19 tumors (3.6\%) were diagnosed as non-GISTs, and the other 93 (18.1\%) GISTs were reclassified into lower risk categories by central pathology. Among 10 patients with non-GISTs and 8 patients with PDGFRA D842V mutations, 4 (40\%) and $3(38 \%)$ patients, respectively, continued the therapy after receiving the central pathology results.

Conclusions The adherence to guidelines and the concordance of pathological diagnoses were comparatively good for highrisk GISTs. Central pathology may contribute to improved diagnosis, but further refinements may be required.
\end{abstract}

Keywords Gastrointestinal stromal tumor $\cdot$ Guidelines $\cdot$ Pathological diagnosis $\cdot$ Multidisciplinary board $\cdot$ Adjuvant therapy

$\begin{array}{ll}\text { Abbreviations } \\ \text { GIST } & \text { Gastrointestinal stromal tumor } \\ \text { QOL } & \text { Quality of life } \\ \text { NCCN } & \text { National Comprehensive Cancer Network } \\ \text { ESMO } & \text { European Society for Medical Oncology } \\ \text { CI } & \text { Confidence interval } \\ \text { PDGFRA } & \text { Platelet-derived growth factor receptor alpha } \\ \text { NIH } & \text { National Institutes of Health }\end{array}$

The list of hospitals and representative investigators is shown in Supplemental Table 5.

Electronic supplementary material The online version of this article (https://doi.org/10.1007/s10120-019-00966-4) contains supplementary material, which is available to authorized users.

Toshirou Nishida

tnishida@ncc.go.jp

Extended author information available on the last page of the article

$\begin{array}{ll}\text { IRB } & \text { Institutional review board } \\ \text { IHC } & \text { Immunohistochemistry } \\ \text { HPF } & \text { High-power field } \\ \text { PS } & \text { Performance status }\end{array}$

\section{Introduction}

Rare cancers present several challenges, including delays in the diagnosis and the administration of treatments that are not always based on the guidelines used in clinical practice [1]. Furthermore, there is significant discordance in the pathological diagnosis of rare cancers between disease-specific pathologists and general pathologists, which rarely occurs for common cancers [2]. Soft tissue sarcoma is a typical rare cancer with a wide variety of histological subtypes originating from connective tissue, bone, and viscera. In sarcoma, major discordance rates of $10-20 \%$ in the 
pathological diagnosis between disease-specific and general pathologists has been reported, and a rate of minor pathological discrepancies, such as different grading, of $20 \%$ has been reported [3-5]. Gastrointestinal stromal tumors (GISTs) are soft tissue sarcomas with established diagnostic criteria based on KIT and DOG1 immunostaining and genotyping [6-8]. The established treatment for GISTs is imatinib, sunitinib or regorafenib after the discovery of driver mutations in the KIT or PDGFRA gene [9]. These facts may indicate that GISTs might differ from other soft tissue sarcomas in terms of the pathological diagnosis and in clinical practice; however, the concordance of the pathological diagnosis of GISTs in the real world is still unknown, and factors related to this pathological discordance have yet to be investigated.

Diagnosis and treatment based on guidelines and sharing the treatment decision with patients under the supervision of a multidisciplinary team are suggested to improve the quality of life (QoL) and prognosis of patients [10]. The first clinical practice guidelines for GISTs were published by the National Comprehensive Cancer Network (NCCN) in 2004 [6], followed by guidelines published by the European Society for Medical Oncology (ESMO) [7], and they have been revised along with the development of innovative medicine. With an increase in the amount of established clinical evidence, each country, including Japan [8], has its own clinical practice guidelines for GISTs. Although many GIST guidelines have been published and revised several times, there are few reports on the clinical use and adherence to the guidelines in clinical practice [11-13]. We prospectively registered patients with high-risk GISTs diagnosed in local hospitals and institutions. Using data obtained from the registry study, we examined the concordance of pathological diagnoses, measured the discordance between the guidelines and clinical practice in terms of adjuvant therapy in the treatment of high-risk GISTs, and investigated potential causes of the discordance.

\section{Patients and methods}

\section{Study design}

Between Dec. 2012 and Dec. 2015, a total of 541 patients with high-risk GISTs who were pathologically diagnosed and treated in each participating hospital were recruited to participate in the prospective observational registry study (STAR ReGISTry) (Supplemental Fig. 1). The modified National Institutes of Health (NIH) consensus criteria were used for risk stratification in this study [14]. As two patients were later revealed to have intermediate-risk GISTs by the local diagnosis, one tumor was a recurrent tumor, and one registration was a duplicate, a total of 537 patients were eligible for registration. In our STAR ReGISTry, we also collected surgical specimens for the central pathology and genotyping of the KIT and PDGFRA genes conducted by one of the authors. In the central pathology, a disease-specific pathologist reviewed all materials and data of hematoxylin and eosin staining, immunohistochemistry staining, and genotyping using obtained surgical specimens. Among the 537 patients, central pathology and genotyping findings were available for 534 patients, as three patients lacked specimens (Supplemental Fig. 1). Ethical approval for the study was initially obtained from the institutional review board (IRB) of the National Cancer Center and then from those of the other participating hospitals. Signed informed consent was obtained from all participating patients. This trial is registered with the UMIN Clinical Trials Registry, number UMIN000009531.

In the central pathological examinations, hematoxylin and eosin (H\&E) staining and immunohistochemistry (IHC) for KIT, DOG1, CD34 and Ki67 were performed as previously described $[9,15]$. The Ki-67 labeling index was calculated as a percentage of Ki-67-positive tumor cells to all tumor cells in approximately 1000 cells of the hot spot. Since the field number of the microscope used in the central review was 26.5, 50 high-power fields (HPFs) corresponded to $17.235 \mathrm{~mm}^{2}$. Genotyping was performed after the extraction of genomic DNA from the paraffin sections using the QIAamp DNA Mini Kit (QIAGEN, Hilden, Germany); exons $9,11,13$, and 17 of the KIT gene and exons 12, 14, and 18 of the PDGFRA gene were amplified by PCR and sequenced as described elsewhere $[9,15]$.

In this research, we also conducted a questionnaire survey in each participating hospital as research accompanying the STAR ReGISTry. The study was approved by the IRB of the Osaka Police Hospital and the National Cancer Center. These studies were conducted in accordance with the World Medical Association Declaration of Helsinki, Ethical Principles for Medical Research Involving Human Subjects (Amended in Seoul in October 2008) and the Ethics Guidelines for Clinical Research (Ministry of Health, Labour and Welfare Notice no. 415, 2008).

\section{Statistical analysis}

The demographics and baseline characteristics of the patients with and without adjuvant therapy were compared, and differences were tested using the Chi-squared test or the Mann-Whitney $U$ test to identify risk factors associated with non-adherence. Multiple logistic regression analysis of the demographics and baseline characteristics as covariates was performed, and variable selection was performed using a stepwise method. The data were analyzed using SAS Version 9.4 (SAS Institute, Inc., Cary, NC, USA). This report is based on baseline data fixed on January 31, 2018. 


\section{Results}

The enrolled patients included 294 (55\%) men and 240 (45\%) women, with a median age of 65 years. GISTs were located in the stomach $(N=318 ; 60 \%)$, small intestine $(163 ; 31 \%)$, colon and rectum $(32 ; 6 \%)$, esophagus $(7 ; 1 \%)$ and extragastrointestinal tissues (14; 3\%) (Table 1). The median tumor size was $7.5 \mathrm{~cm}$, and the median mitotic count was $10 / 50$ HPFs, as determined by the diagnosis at local hospitals. Tumor rupture, including 33 preoperative ruptures and 29 intraoperative ruptures, was observed in $66(12 \%)$ patients. Histologically, the tumors comprised $446(84 \%)$ spindle cell-type tumors, 17 (3\%) epithelioid cell-type tumors and 46 (9\%) mixed cell-type tumors. Open surgery was performed in 387 (72\%) cases, and laparoscopic surgery was performed in 147 (28\%) cases. Clinically significant morbidities due to surgery were observed in 63 patients, and most were less than grade 2 (Supplemental Table 1). No deaths related to surgery occurred.

Of 534 patients, $432(81 \%)$ received imatinib adjuvant therapy. The starting dose of imatinib was $400 \mathrm{mg}$ /day for 314 patients and $300 \mathrm{mg} /$ day for 77 patients. The other 102 patients did not undergo adjuvant therapy due to a fear of adverse events due to imatinib $(N=39 ; 38 \%)$, economic reasons $(26 ; 26 \%)$, advanced age $(20 ; 20 \%)$, patient refusal for unidentifiable reasons $(20 ; 20 \%)$, and comorbidities $(15 ; 15 \%)$, as shown in Table 2 . Some patients and investigators doubted the evidence of improvements in overall survival (OS) due to adjuvant therapy and were concerned about the potential induction of drug resistance by the therapy. Patients without adjuvant therapy were older, exhibited poorer performance statuses (PSs), and more frequently had nongastric GISTs (Table 3 ). The GISTs of patients with adjuvant therapy were larger, showed a higher mitotic count, and ruptured more frequently than those of patients without adjuvant therapy. Multivariate analysis indicated that age (HR $0.71 ; 95 \%$ CI $0.58-0.88$; $P=0.002$ ), tumor size (HR for $>10 \mathrm{~cm} \mathrm{vs}<5 \mathrm{~cm}$, $3.87 ; 95 \%$ CI $1.72-8.74 ; P=0.001)$, mitotic count (HR for $>10 / 50 \mathrm{HPFs}$ vs $<5 / 50,3.54$; $95 \%$ CI $1.84-6.79$; $P<0.001$ ), tumor rupture (HR 3.69; 95\% CI 1.43-9.52; $P=0.007)$ and PS (HR $0.55 ; 95 \%$ CI $0.31-0.99 ; P=0.046)$ were independent factors of the administration of adjuvant therapy (Supplemental Table 2).

Next, we evaluated pathological concordance for high-risk GISTs between local, general pathologists and a central GIST specialist and surveyed the influence of the results of the central pathological examination on treatment changes. Of 534 GISTs that had been locally diagnosed as high-risk GISTs, 19 tumors $(3.6 \%)$ were diagnosed as non-GISTs in central pathology (Table 4; the pathological features of which are summarized in
Table 1 Patients characteristics

\begin{tabular}{|c|c|}
\hline Total & $(N=534)$ \\
\hline Age (median, IQR; years) & $65(56-72)$ \\
\hline \multicolumn{2}{|l|}{ Gender } \\
\hline Male & $294(55 \%)$ \\
\hline Female & $240(45 \%)$ \\
\hline \multicolumn{2}{|l|}{ PS } \\
\hline 0 & $447(84 \%)$ \\
\hline 1 & $76(14 \%)$ \\
\hline 2 & $4(1 \%)$ \\
\hline 3 & $3(1 \%)$ \\
\hline Unavailable & $4(1 \%)$ \\
\hline \multicolumn{2}{|l|}{ Location } \\
\hline Esophagus & $7(1 \%)$ \\
\hline Stomach & $318(60 \%)$ \\
\hline Small intestine & $163(31 \%)$ \\
\hline Colon and rectum & $32(6 \%)$ \\
\hline Others & $14(3 \%)$ \\
\hline \multicolumn{2}{|l|}{ Neoadjuvant therapy } \\
\hline$(-)$ & $475(89 \%)$ \\
\hline$(+)$ & $59(11 \%)$ \\
\hline \multicolumn{2}{|l|}{ Surgery } \\
\hline Open & $387(72 \%)$ \\
\hline Laparoscopic & $147(28 \%)$ \\
\hline \multicolumn{2}{|l|}{ Curability of surgery } \\
\hline R0 & $517(97 \%)$ \\
\hline $\mathrm{R} 1$ & $17(3 \%)$ \\
\hline Tumor size $(\mathrm{cm} ; n=533)$ & $\begin{array}{l}7.5(5.5-11.3) \\
\text { (median, } \\
\text { IQR) }\end{array}$ \\
\hline Unknown & 1 \\
\hline \multicolumn{2}{|c|}{ Mitosis (/50HPF; $n=497)$ at local $10(5-23)$ (median, IQR) } \\
\hline Unknown & 38 \\
\hline \multicolumn{2}{|l|}{ Tumor rupture } \\
\hline No & $459(86 \%)$ \\
\hline Yes & $66(12 \%)$ \\
\hline Preoperative & $33(6 \%)$ \\
\hline Intraoperative & $29(5 \%)$ \\
\hline Unknown & $9(2 \%)$ \\
\hline \multicolumn{2}{|l|}{ Histological types } \\
\hline Spindle & $446(84 \%)$ \\
\hline Epithelioid & $17(3 \%)$ \\
\hline Mixed & $46(9 \%)$ \\
\hline Unavailable & $25(5 \%)$ \\
\hline \multicolumn{2}{|l|}{ Genotyping } \\
\hline KIT & $457(86 \%)$ \\
\hline PDGFRA & $18(3 \%)$ \\
\hline Wild type & $36(7 \%)$ \\
\hline Unavailable & $22(4 \%)$ \\
\hline
\end{tabular}


Table 2 Reasons for no adjuvant therapy

\begin{tabular}{ll}
\hline Reasons for no adjuvant therapy & Total $N=102(\%)$ \\
\hline Fear of adverse events of imatinib & $39(38.2 \%)$ \\
Economic reasons & $26(25.5 \%)$ \\
Advanced age & $20(19.6 \%)$ \\
Patient refusal unknown & $20(19.6 \%)$ \\
Comorbidities & $15(14.7 \%)$ \\
Doubts for evidences of OS & $10(9.8 \%)$ \\
Re-review results of central pathology & $3(2.9 \%)$ \\
Fear of resistant mutations & $2(2.0 \%)$ \\
Poor PS & $1(1.0 \%)$ \\
Others & $2(2.0 \%)$ \\
\hline
\end{tabular}

Supplemental Table 3). The non-GISTs showed unusual locations, including extragastrointestinal locations, negative staining for KIT and DOG1 in the central pathological diagnosis, and no mutations in the KIT and PDGFRA genes (Supplemental Tables 3 and 4). Of 515 centrally certified GISTs, 93 (18.1\%) GISTs were reclassified into lower categories due to different mitotic counts in the central pathological diagnosis (Table 4). While the local and central mitotic counts were well correlated, the former were significantly higher than the latter (Supplemental Fig. 2).

Since the guidelines do not recommend imatinib adjuvant therapy for non-GISTs or for GISTs with PDGFRA D842V mutations, which are considered to be resistant to all available tyrosine kinase inhibitors, including imatinib, we used the questionnaire to examine changes in the clinical practice of local hospitals after receiving the central pathology results (Table 5). Of 19 non-GIST patients in the central pathological diagnosis, 10 patients who were undergoing imatinib adjuvant therapy at the time the results were returned were evaluable. Six patients $(60 \%)$ stopped imatinib adjuvant therapy, and the other 4 continued the therapy despite being diagnosed as having a non-GIST by central pathology. Similarly, 8 patients with GISTs with PDGFRA D842V mutations were evaluable; $5(63 \%)$ patients stopped adjuvant therapy after the central pathology results were reported, and the other three continued. Central pathological review and genetic analysis results were shared with all patients who have had treatment changes to discontinue adjuvant therapy, however, central reports were not always shared in the other cases. Some of the latter patients had already stopped adjuvant therapy before central reporting due to disease relapses or adverse events. Among patients with wild-type GISTs, all 7 evaluable patients continued imatinib adjuvant therapy after the reporting. However, almost all investigators participating in this study answered that they always refer to the GIST guidelines in their clinical practice and that they treat GIST patients according to the guidelines (Supplemental Table 3).

\section{Discussion}

Diagnosis and treatment according to the clinical practice guidelines and specialized multidisciplinary management have been indicated to be associated with improved QoL and survival $[1,10,16]$, especially in rare cancers. Among GISTs, high-risk GISTs are considered to require a multidisciplinary approach, such as adjuvant therapy, to improve the prognostic outcome [6-8]. In this study, we examined the adherence to the guidelines and the concordance of the pathological diagnosis in the real world using data obtained from a prospective registry of high-risk GISTs with central pathology. Two-thirds of participating hospitals reported, on average, annual experiences of less than 10 primary GISTs/year (Supplemental Fig. 3), suggesting that the study may include standard acute care hospitals in Japan. The study indicated that adjuvant therapy was performed for $81 \%$ of high-risk GIST patients diagnosed locally. This value appears to be slightly better than those in previous reports from the USA and Canada [11-13, 17], in which adjuvant therapy was performed for $68 \%$ of high-risk GIST patients and for nearly $70 \%$ in high-volume centers. The difference may be due to the different timeframe of our study (2012-2015) and the American studies (2004-2009 for Pisters [11], 2006-2007 for Bilimoria [12], or 2009-2012 for Bischof [13]). In fact, the proportion of patients receiving adjuvant therapy has increased over time. Bilimoria et al. [12] indicated that adjuvant therapy was more likely to be administered to a patient with a large tumor $(>6 \mathrm{~cm})$, a high-grade tumor, and a positive marginal status at a high-volume cancer center in a recent time period. Bischof et al. [17] indicated that the tumor size, mitotic rate, and neoadjuvant therapy were independent determinants of adjuvant therapy after adjusting for confounding variables. In our study, the tumor size, mitotic rate, and tumor rupture were found to be positive selection factors for adjuvant therapy, while age and PS were negative selection factors. The reported reasons for not undergoing adjuvant therapy are reasonable, and taken together, these findings indicate that treatment options are generally shared and selected with patients according to their conditions and intention in the real world.

Previously, pathological diagnosis has been suggested to not always be consistent among pathologists, even in clinical trials $[2,18]$. The inconsistency among pathologists is greater for rare cancers. For sarcomas, several retrospective studies have found a major discordance rate of more than $10 \%$ between sarcoma pathologists and general pathologists [3-5], which may result in delayed diagnosis and poor patient outcomes [1]. Contrary to soft tissue sarcomas, 
Table 3 Clinicopathological features of adjuvant and nonadjuvant patients

\begin{tabular}{|c|c|c|c|}
\hline Total & Non-adjuvant $(N=102)$ & Adjuvant $(N=432)$ & $P$ value \\
\hline Age (median, IQR; years) & $70.5(60-80)$ & $64(55-71)$ & $<0.001$ \\
\hline \multicolumn{4}{|l|}{ Gender } \\
\hline Male & $58(56.9 \%)$ & $236(54.6 \%)$ & \multirow[t]{2}{*}{0.766} \\
\hline Female & $44(43.1 \%)$ & $196(45.4 \%)$ & \\
\hline \multicolumn{4}{|l|}{ Location } \\
\hline Esophagus & $5(4.9 \%)$ & $2(0.5 \%)$ & \multirow[t]{5}{*}{0.005} \\
\hline Stomach & $56(54.9 \%)$ & $262(60.6 \%)$ & \\
\hline Small intestine & $30(29.4 \%)$ & $133(30.8 \%)$ & \\
\hline Colon and rectum & $9(8.8 \%)$ & $23(5.3 \%)$ & \\
\hline Others & $2(2.0 \%)$ & $12(2.8 \%)$ & \\
\hline \multicolumn{4}{|l|}{ PS } \\
\hline 0 & $77(75.5 \%)$ & $370(85.6 \%)$ & \multirow[t]{4}{*}{0.004} \\
\hline 1 & $20(19.6 \%)$ & $56(13.0 \%)$ & \\
\hline 2 & $3(2.9 \%)$ & $1(0.2 \%)$ & \\
\hline 3 & $2(2.0 \%)$ & $1(0.2 \%)$ & \\
\hline \multicolumn{4}{|l|}{ Neoadjuvant } \\
\hline$(-)$ & $95(93.1 \%)$ & $380(88.0 \%)$ & \multirow[t]{2}{*}{0.186} \\
\hline$(+)$ & $7(6.9 \%)$ & $52(12.0 \%)$ & \\
\hline \multicolumn{4}{|l|}{ Surgery } \\
\hline Open & $71(69.6 \%)$ & $316(73.1 \%)$ & \multirow[t]{2}{*}{0.551} \\
\hline Laparoscopic & $31(30.4 \%)$ & $116(26.9 \%)$ & \\
\hline \multicolumn{4}{|l|}{ Curability of surgery } \\
\hline R0 & $100(98.0 \%)$ & $417(96.5 \%)$ & \multirow[t]{2}{*}{0.639} \\
\hline $\mathrm{R} 1$ & $2(2.0 \%)$ & $15(3.5 \%)$ & \\
\hline \multicolumn{4}{|c|}{ Tumor size (median, IQR: cm) } \\
\hline & $6.5(5.1-10.0)$ & $7.7(5.5-12.0)$ & \multirow[t]{2}{*}{0.035} \\
\hline Unavailable & 0 & 1 & \\
\hline \multicolumn{4}{|c|}{ Mitosis (median, IQR:/50HPF) at local } \\
\hline & $8(4-17)$ & $11(5-25)$ & \multirow[t]{2}{*}{0.005} \\
\hline Unavailable & 6 & 31 & \\
\hline \multicolumn{4}{|l|}{ Tumor rupture } \\
\hline No & $94(92.2 \%)$ & $365(84.5 \%)$ & \multirow[t]{3}{*}{0.042} \\
\hline Yes & $6(5.9 \%)$ & $60(13.9 \%)$ & \\
\hline Unavailable & $2(2.0 \%)$ & $7(1.6 \%)$ & \\
\hline \multicolumn{4}{|l|}{ Histological types } \\
\hline Spindle & $86(84.3 \%)$ & $360(83.3 \%)$ & \multirow[t]{4}{*}{0.343} \\
\hline Epithelioid & $1(1.0 \%)$ & $16(3.7 \%)$ & \\
\hline Mixed & $10(9.8 \%)$ & $36(8.3 \%)$ & \\
\hline Unavailable & $5(4.9 \%)$ & $20(4.6 \%)$ & \\
\hline \multicolumn{4}{|l|}{ Genotyping } \\
\hline KIT & $84(82.4 \%)$ & $372(86.1 \%)$ & \multirow[t]{4}{*}{0.829} \\
\hline PDGFRA & $4(3.9 \%)$ & $14(3.2 \%)$ & \\
\hline Wild type & $8(7.8 \%)$ & $29(6.7 \%)$ & \\
\hline Unavailable & $10(9.8 \%)$ & $31(7.2 \%)$ & \\
\hline
\end{tabular}

GISTs may not show such a high major discordance rate; for GISTs, the diagnostic criteria are indicated by the guidelines [6-8, 19]. Our major discordance rate for high-risk GISTs $(3.6 \%)$ appears to be better than that in European reports, in which pathological concordance according to the tumor category is $90.6 \%$ and $87.0 \%$ for GISTs and soft tissue sarcomas, respectively $[5,20]$. Our study indicates that key factors of concordance may include positive KIT and DOG1 staining and genotyping and that special care may be required for extragastrointestinal GISTs. In this study, 
Table 4 Concordance between local and central diagnosis

\begin{tabular}{lcr}
\hline Central pathology & Pts no. $(N=534)$ & $\%$ \\
\hline Histology & & \\
Non-GIST & 515 & 3.6 \\
GIST & & 96.4 \\
Risk re-classification of true GISTs & \\
Risk classification in the central pathology $(N=515)$ & \\
High risk & 411 & 79.8 \\
Intermediate & 64 & 12.4 \\
Low & 25 & 4.9 \\
Very low & 4 & 0.8 \\
\hline
\end{tabular}

we observed a minor discordance rate of $17.4 \%$ in the risk classification, which was caused by different mitotic rates between local and central pathologists. Thus, in total, nearly $20 \%$ of discordance may exist between general and diseasespecific pathologists. The local and central mitotic counts of pathologists were well correlated; however, the former were significantly higher than the latter. This may be caused by differences in the microscopic fields and sections between the two pathologists, the field number of each microscope, and mitotic figure counts. The field number of the central microscope was one of the widest, as mentioned in the "Patients and methods" section. Most sections submitted for central pathology likely originate from areas similar to those used for local diagnosis, and mitotic differences caused by differences in the paraffin sections may disappear after the accumulation of sufficient numbers. Thus, different mitotic counts between pathologists may be speculated to be causative; however, we did not have a central review of GISTs other than high-risk tumors in this study, so we could not identify the true causes of the differences between the local and central mitotic counts.

Practical discordance in the pathological diagnosis of rare cancers may be resolved by central pathology or pathological consultation [18]. No reports, however, have tracked and evaluated the treatment changes in local hospitals after the return of the central pathology report, including the genotype. We found that for both patients with non-GISTs and GISTs with the PDGFRA D842V mutation, $40 \%$ of patients continued imatinib adjuvant therapy, although the guidelines do not recommend the therapy and local investigators themselves reported that their clinical practices comply with the guidelines, suggesting that central pathology results may not always be used locally. Interestingly, adjuvant therapy was continued in wild-type GIST after reporting central pathology as the guidelines suggest. This study examined a limited number of Japanese patients; however, it may be speculated that similar phenomena may occur in western countries and other Asian countries. In fact, discordance in pathological diagnosis appears to be universal in rare cancer as mentioned above. Taken together, the results suggest that in addition to central pathology, other methods might be required to facilitate treatment changes in local hospitals.

The study has several limitations. As adherence to the guidelines was high and the pathological discordance was small in our series, the power of the statistical analysis may not be sufficient to identify factors contributing to pathological discordance or guideline adherence. We only examined clinical practice in Japan. These phenomena should be evaluated in an international large-scale study with real-world data. Our study is based on a prospective registry involving general hospitals in Japan, and the data are nearly complete, with a high follow-up rate. Since this study used the fixed baseline data of the registry study, we cannot evaluate the effects of treatment adherence, adjuvant therapy completion rate or prognostic outcome of patients with high-risk GISTs with or without adjuvant therapy, which may require at least another 2 years.

In summary, we have been maintaining a prospective registry of high-risk GISTs and analyzed the baseline data along with the results of a questionnaire survey administered to participating investigators. In the registry study, adjuvant therapy was administered to $81 \%$ of high-risk GIST patients in the real world. The reasons some patients did not undergo adjuvant therapy were a fear of adverse events, economic reasons, advanced age and comorbidities. In the study, the
Table 5 Changes in adjuvant therapy after central pathology

\begin{tabular}{llll}
\hline & Non-GIST $(N=19)$ & $\begin{array}{l}\text { PDFGRA exon18 } \\
\text { D842V }(N=17)\end{array}$ & Wild type $(N=19)$ \\
\hline Initially no adjuvant therapy & 5 & 3 & 4 \\
No. of patients received adjuvant & 14 & 14 & 15 \\
Unevaluable due to other reasons ${ }^{\mathrm{a}}$ & 4 & 4 & 8 \\
No. of evaluable patients with adjuvant & 10 & 8 & 7 \\
Stopped by central pathology & $6(60 \%)$ & $5(63 \%)$ & $0(0 \%)$ \\
Continued after central pathology & $4(40 \%)$ & $3(38 \%)$ & $7(100 \%)$ \\
\hline
\end{tabular}

${ }^{\mathrm{a}}$ Imatinib adjuvant therapy was already stopped before returning central pathology due to relapses or patients' refusal of imatinib due to adverse events 
rates of major and minor discordance in the pathological diagnosis of GISTs were $3.6 \%$ and $17.4 \%$, respectively, and these values are better than those observed for soft tissue sarcomas. Although central pathology and genotyping may fill the gap between general and specialized pathology, further improvements and other methods may be required to improve clinical practice in general hospitals.

Acknowledgements The authors are grateful to all participating investigators listed in Supplement Table 5.

Author contributions Conception and design: TN, YK, YK, TM, and YN. Administrative support: TK. Provision of study materials or patients: TN, YS, MT, MO, YK, YK, TM, YN, and SH. Collection and assembly of data: all the authors. Data analysis and interpretation: TN, TK, and SH. Manuscript writing: all the authors. Final approval of manuscript: all the authors.

Funding The STAR ReGISTry Study itself is mainly supported by Novartis Pharma. A part of this work including research accompanying the STAR ReGISTry is supported in part by a Grant (28-A-16) from the National Cancer Center Research and Development Fund.

\section{Compliance with ethical standards}

Conflict of interest Yuko Kitagawa, Toru Masuzawa, Tatsuo Kagimura, and Masakazu Takagi have no COI in terms of Employment, Leadership, Stock and Other Ownership Interests, Honoraria, Consulting or Advisory Role, Speakers' Bureau, Research Funding, Patents, Royalties, Other Intellectual Property, Expert Testimony, Travel, Accommodations, Expenses and Other Relationship. Toshirou Nishida has been paid Honoraria by Pfizer, Bayer, Eizai, and Taiho. Seiichi Hirota has been paid Honoraria by Novartis and Pfizer. Yoichi Naito has Consulting or Advisory Role for Pfizer, Eisai, Eli Lilly, Chugai, Novartis, and AstraZeneca, Speakers' Bureau of Pfizer, Novartis, Chugai, Eisai, AstraZeneca, Shionogi, Hisamitus, Fuji RI farma, JSMO, and Technical Information Institute, and Research Funding form AstraZeneca, Eli Lilly, Pfizer, Ono, Taiho, CSPOR-BC, and JBCRG. Yukinori Kurokawa has been paid Honoraria by Taiho, Eli Lilly, Yakult Honsha, Ono, MSD, Kaken Pharmaceutical, Medtronic, Johnson \& Johnson, and Stryker. Yoshiharu Sakai has Speakers' Bureau of Taiho, Chugai, Tsumura, Yakult, Sanofi, and MSD, and Research Funding from Taiho, Chugai, Yaklut, and Sanofi. Masato Ozaka has been paid Honoraria by Taiho, Yakult, Novartis, Bayer, Pfizer, and Takeda, and has Research Funding from Baxalta, Taiho, Incyte, and Boston biomedical. The others have no COI to declare.

Open Access This article is distributed under the terms of the Creative Commons Attribution 4.0 International License (http://creativeco mmons.org/licenses/by/4.0/), which permits unrestricted use, distribution, and reproduction in any medium, provided you give appropriate credit to the original author(s) and the source, provide a link to the Creative Commons license, and indicate if changes were made.

\section{References}

1. Ray-Coquard I, Pujade Lauraine E, Le Cesne A, Pautier P, Vacher Lavenue MC, Trama A, et al. Improving treatment results with reference centres for rare cancers: where do we stand? Eur J Cancer. 2017;77:90-8.
2. Gilchrist KW, Harrington DP, Wolf BC, Neiman RS. Statistical and empirical evaluation of histopathologic reviews for quality assurance in the Eastern Cooperative Oncology Group. Cancer. 1988;62:861-8.

3. Lurkin A, Ducimetière F, Vince DR, Decouvelaere AV, Cellier D, Gilly FN, et al. Epidemiological evaluation of concordance between initial diagnosis and central pathology review in a comprehensive and prospective series of sarcoma patients in the Rhone-Alpes region. BMC Cancer. 2010;10:150. https://doi. org/10.1186/1471-2407-10-150.

4. Ray-Coquard I, Montesco MC, Coindre JM, Dei Tos AP, Lurkin A, et al. Sarcoma: concordance between initial diagnosis and centralized expert review in a population-based study within three European regions. Ann Oncol. 2012;23:2442-9.

5. Thway K, Wang J, Mubako T, Fisher C. Histopathological diagnostic discrepancies in soft tissue tumours referred to a specialist centre: reassessment in the era of ancillary molecular diagnosis. Sarcoma. 2014;2014:686902. https://doi.org/10.1155/2014/68690 2

6. Demetri GD, von Mehren M, Antonescu CR, DeMatteo RP, Ganjoo KN, Maki RG, et al. NCCN Task Force report: update on the management of patients with gastrointestinal stromal tumors. J Natl Compr Canc Netw. 2010;8(Suppl 2):S1-41.

7. Casali PG, Abecassis N, Bauer S, Biagini R, Bielack S, Bonvalot $\mathrm{S}$, et al. Gastrointestinal stromal tumours: ESMO-EURACAN Clinical Practice Guidelines for diagnosis, treatment and followup. Ann Oncol. 2018;29(Supplement_4):iv68-iv78.

8. Nishida T, Hirota S, Yanagisawa A, Sugino Y, Minami M, Yamamura $\mathrm{Y}$, et al. Clinical practice guidelines for gastrointestinal stromal tumor (GIST) in Japan: English version. Int J Clin Oncol. 2008;13:416-30.

9. Hirota S, Isozaki K, Moriyama Y, Hashimoto K, Nishida T, Ishiguro S, et al. Gain-of-function mutations of c-kit in human gastrointestinal stromal tumors. Science. 1998;279(5350):577-80.

10. Blay JY, Soibinet P, Penel N, Bompas E, Duffaud F, Stoeckle E, et al. Improved survival using specialized multidisciplinary board in sarcoma patients. Ann Oncol. 2017;28:2852-9.

11. Pisters PW, Blanke CD, von Mehren M, Picus J, Sirulnik A, Stealey E, et al. A USA registry of gastrointestinal stromal tumor patients: changes in practice over time and differences between community and academic practices. Ann Oncol. 2011;22:2523-9.

12. Bilimoria KY, Wayne JD, Merkow RP, Abbott DE, Cormier JN, Feig BW, et al. Incorporation of adjuvant therapy into the multimodality management of gastrointestinal stromal tumors of the stomach in the United States. Ann Surg Oncol. 2012;19:184-91.

13. Bischof DA, Kim Y, Blazer DG 3rd, Behman R, Karanicolas PJ, Law $\mathrm{CH}$, et al. Surgical management of advanced gastrointestinal stromal tumors: an international multi-institutional analysis of 158 patients. J Am Coll Surg. 2014;219:439-49.

14. Joensuu H, Vehtari A, Riihimäki J, Nishida T, Steigen SE, Brabec $\mathrm{P}$, et al. Risk of recurrence of gastrointestinal stromal tumour after surgery: an analysis of pooled population-based cohorts. Lancet Oncol. 2012;13:265-74.

15. Hirota S, Ohashi A, Nishida T, Taniguchi M, Nishikawa K, Ohashi A, et al. Gain-of-function mutations of platelet-derived growth factor receptor alpha gene in gastrointestinal stromal tumors. Gastroenterology. 2003;125:660-7.

16. Rossi CR, Vecchiato A, Mastrangelo G, Montesco MC, Russano $\mathrm{F}$, Mocellin $\mathrm{S}$, et al. Adherence to treatment guidelines for primary sarcomas affects patient survival: a side study of the European CONnective TIssue CAncer NETwork (CONTICANET). Ann Oncol. 2013;24:1685-91.

17. Bischof DA, Dodson R, Jimenez MC, Behman R, Cocieru A, Blazer DG 3rd, et al. Adherence to guidelines for adjuvant imatinib therapy for GIST: a multi-institutional analysis. J Gastrointest Surg. 2015;19:1022-8. 
18. Gupta D, Layfield LJ. Prevalence of inter-institutional anatomic pathology slide review: a survey of current practice. Am J Surg Pathol. 2000;24:280-4.

19. Dorfman DM, Bui MM, Tubbs RR, Hsi ED, Fitzgibbons PL, Linden MD, et al. The CD117 immunohistochemistry tissue microarray survey for quality assurance and interlaboratory comparison: a College of American Pathologists Cell Markers Committee Study. Arch Pathol Lab Med. 2006;130:779-82.

20. Perrier L, Rascle P, Morelle M, Toulmonde M, Ranchere Vince $\mathrm{D}$, et al. The cost-saving effect of centralized histological reviews with soft tissue and visceral sarcomas, GIST, and desmoid tumors: The experiences of the pathologists of the French Sarcoma Group. PLoS One. 2018;13:e0193330. https://doi.org/10.1371/journ al.pone. 0193330 .

Publisher's Note Springer Nature remains neutral with regard to jurisdictional claims in published maps and institutional affiliations.

\section{Affiliations}

\section{Toshirou Nishida ${ }^{1}$ - Yoshiharu Sakai ${ }^{2} \cdot$ Masakazu Takagi $^{3} \cdot$ Masato Ozaka $^{4} \cdot$ Yuko Kitagawa $^{5} \cdot$ Yukinori Kurokawa $^{6}$.

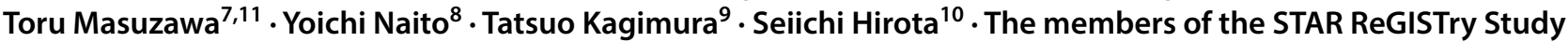 Group}

1 Department of Surgery, National Cancer Center Hospital, 5-1-1 Tsukiji, Chuoku, Tokyo 104-0045, Japan

2 Department of Surgery, Kyoto University Hospital, Kyoto, Japan

3 Department of Gastroenterological Surgery, Shizuoka General Hospital, Shizuoka, Japan

4 Department of Gastroenterological Medicine, Cancer Institute Hospital Japanese Foundation for Cancer Research, Tokyo, Japan

5 Department of Surgery, Keio University Hospital, Tokyo, Japan

6 Department of Gastroenterological Surgery, Osaka University Graduate School of Medicine, Suita, Japan
7 Department of Surgery, Osaka Police Hospital, Osaka, Japan

8 Department of Breast and Medical Oncology, National Cancer Center Hospital East, Kashiwa, Japan

9 Translational Research Center for Medical Innovation, Foundation for Biomedical Research and Innovation at Kobe, Kobe, Japan

10 Department of Surgical Pathology, Hyogo College of Medicine, Nishinomiya, Japan

11 Present Address: Department of Surgery, Kansai Rosai Hospital, Amagasaki, Japan 